\title{
Erratum to: Biogeographically interesting planktonic Nostocales (Cyanobacteria) in the Czech Republic and their polyphasic evaluation resulting in taxonomic revisions of Anabaena bergii Ostenfeld 1908 (Chrysosporum gen. nov.) and A. tenericaulis Nygaard 1949 (Dolichospermum tenericaule comb. nova)
}

\author{
Eliška Zapomělová • Olga Skácelová • \\ Petr Pumann • Radovan Kopp • Emil Janeček
}

Published online: 29 March 2013

(C) Springer Science+Business Media Dordrecht 2013

In the above mentioned publication, the new combinations Chrysosporum ovalisporum (Forti) comb. nov. and Dolichospermum tenericaule comb. nov. were invalid, as the names of the basionyms and the pages of related publications were not cited in accordance

The online version of the original article can be found under doi:10.1007/s10750-012-1034-z.

E. Zapomělová $(\bowtie)$

Biology Centre of AS CR, Institute of Hydrobiology, Na Sádkách 7, 37005 České Budějovice, Czech Republic e-mail: eliska.zapomelova@seznam.cz

O. Skácelová

Faculty of Science, University of South Bohemia, Branišovská 31, 37005 České Budějovice,

Czech Republic

P. Pumann

National Institute of Public Health, Šrobárova 48, 10042 Praha 10, Czech Republic

\section{R. Kopp}

Department of Fishery and Hydrobiology, Mendel

University in Brno, 61300 Brno, Czech Republic

E. Janeček

Povodí Ohře s. p., Novosedlická 758, 41501 Teplice,

Czech Republic

with the requirements of Article 41.5 of the International Code of Nomenclature for Algae, Fungi and Plants (McNeill et al., 2012).

The authors regret this mistake and therefore validate these combinations as follows:

Chrysosporum ovalisporum (Forti) Zapomělová, Skácelová, Pumann, Kopp \& Janeček comb. nov. [basionym: Aphanizomenon ovalisporum Forti, Atti e Memorie dell' Accademia di Agricoltura, Scienze e Lettere, Arti e Commercio di Verona, Serie 4, vol. 12: 3, 1911 (Forti, 1911)].

Dolichospermum tenericaule (Nygaard) Zapomělová, Skácelová, Pumann, Kopp \& Janeček comb. nov. [basionym: Anabaena tenericaulis Nygaard, Det Kongelige Danske Videnskabernes Selskab, Biologiske Skrifter vol. 7 (1): 207, 1950 (Nygaard, 1950)].

Acknowledgments We thank Prof. Michael Guiry (Algaebase, National University of Ireland) for helpful remarks to this text.

\section{References}

Forti, A., 1911. Diagnoses myxophycearum novarum. Atti e Memorie dell'Accademia di Agricoltura, Scienze e Lettere, Arti e Commercia di Verona, Serie IV 12: 1-5.

McNeill, J., F. R. Barrie, W. R. Buck, V. Demoulin, W. Greuter, D. L. Hawksworth, P. S. Herendeen, S. Knapp, J. Prado, W. 
F. Prud'homme van Reine, G. F. Smith, J. H. Wiersema \& N. J. Turland, 2012. International Code of Nomenclature for algae, fungi and plants (Melbourne Code), adopted by the Eighteenth International Botanical Congress Melbourne, Australia, July 2011. Regnum Vegetabile, Vol. 154. Koeltz Scientific Books, Königstein: 208 pp.

Nygaard, G., 1950 (1949). Hydrobiological studies on some Danish ponds and lakes. Part II: The quotient hypothesis and some new or little known phytoplankton organisms. Det Kongelige Danske Videnskabernes Selskab, Biologiske Skrifter, Vol. 7(1). I. Komission Hos Ejnar Munksgaard, København: 1-294. 